\title{
Determinants and impact of the natriuretic response to diuretic therapy in heart failure with reduced ejection fraction and volume overload
}

\author{
Frederik H. VERBRUGGE ${ }^{1,2}$, MD; Matthias DUPONT ${ }^{1}$, MD; Philippe B. BERTRAND ${ }^{1,2}$, MD, MSc; \\ Petra NIJST ${ }^{1,2}$, MD; Joris PENDERS ${ }^{3}, \mathrm{MD}, \mathrm{PhD}$; Joseph DENS ${ }^{1,4}, \mathrm{MD}, \mathrm{PhD}$; David VERHAERT ${ }^{1}, \mathrm{MD}^{\text {; }}$ \\ Pieter VANDERVOORT ${ }^{1,4}$, MD; W.H. Wilson TANG ${ }^{5}, \mathrm{MD}$; Wilfried MULLENS ${ }^{1,4}, \mathrm{MD}, \mathrm{PhD}$ \\ ${ }^{1}$ Dept. of Cardiology, Ziekenhuis Oost-Limburg, Genk, Belgium; ${ }^{2}$ Doctoral School for Medicine and Life Sciences, Hasselt University, \\ Diepenbeek, Belgium; ${ }^{3}$ Dept. of Laboratory Medicine, Ziekenhuis Oost-Limburg, Genk, Belgium; ${ }^{4}$ Biomedical Research Institute, \\ Faculty of Medicine and Life Sciences, Hasselt University, Diepenbeek, Belgium; ${ }^{5}$ Dept. of Cardiovascular Medicine, \\ Heart and Vascular Institute, Cleveland Clinic, Cleveland, $\mathrm{OH}$, United States of America.
}

Objective The objective of this study was to investigate determinants of the natriuretic response to diuretics in decompensated heart failure (HF) and the relationship with decongestion, neurohumoral activation and clinical outcome in the contemporary era of HF management.

Methods and results In this prospective, single-centre cohort study, consecutive patients with decompensated HF $(n=54)$ and left ventricular ejection fraction $\leq 45 \%$ received protocol-driven diuretic therapy until complete disappearance of congestion signs. Urine was collected during three consecutive 24-h intervals. Natriuretic response was defined as absolute natriuresis (mmol) per $\mathrm{mg}$ of intravenous bumetanide administered. Natriuresis was $146 \mathrm{mmol}(76-206 \mathrm{mmol}), 74 \mathrm{mmol}(37-167 \mathrm{mmol})$ and $74 \mathrm{mmol}(53-134 \mathrm{mmol})$ per $\mathrm{mg}$ intravenous bumetanide administered during the first, second and third 24-h interval, respectively. Diastolic blood pressure $(\beta=23.048 \pm 10.788 ; P$-value $=0.036)$, plasma aldosterone $(\beta=-25.722 \pm 11.560 ; P$-value $=0.029)$, and combination therapy with acetazolamide $(\beta=103.241 \pm 40.962 ; P$-value $=0.014)$ were independent predictors of the natriuretic response. Patients with a stronger natriuretic response demonstrated more pronounced decreases in plasma NT-proBNP levels ( $P$-value $=0.025)$, while a weaker response was associated with higher peak plasma aldosterone levels $(P$-value $=0.013$ ) and plasma renin activity ( $P$-value $=0.033$ ). Natriuresis per loop diuretic dose predicted freedom from all-cause mortality or $\mathrm{HF}$ readmissions, independently of baseline renal function (HR 0.40, 95\%Cl 0.16-0.98; $P$-value $=0.045)$.

Conclusions More effective natriuresis in decompensated HF patients with reduced ejection fraction and volume overload is associated with better decongestion, less neurohumoral activation and predicts favourable clinical outcome independently from renal function per se. Acetazolamide warrants further evaluation in large prospective trials to increase the natriuretic response to loop diuretics.

Keywords Acetazolamide-congestion-diuretics-natriuresis.

\section{INTRODUCTION}

Signs and symptoms of congestion are the main reason for hospital admissions among patients with heart

Address for correspondence:

Frederik Verbrugge, M.D.,

Dept. of Cardiology, Ziekenhuis Oost-Limburg, chiepse Bos 6, 3600 Genk, Belgium.

E-mail: frederik.verbrugge@zol.be

Received 21 October 2014; revision accepted for publication 31 March 2015 failure $(\mathrm{HF})^{1}$. Decongestive treatment in such cases may comprise different strategies, but intravenous loop diuretics remain by far the most frequently applied therapy with $88 \%$ of patients receiving them in the Acute Decompensated Heart Failure National Registry (ADHERE) ${ }^{2}$. The primary objective of diuretics is to achieve a negative sodium balance to reduce extracellular fluid overload and hence signs and symptoms of congestion. However, there are several reasons why the natriuretic response to diuretics might be impaired in HF, especially when renal function is concomitantly depressed. Impaired intestinal absorption of oral therapy because of gut congestion, decreased tubular secretion if renal perfusion is compromised, hypertrophy of the distal 
nephron with increased aldosterone production after prolonged use of loop diuretics, excessive sodium reabsorption in the proximal tubules, and post-diuretic sodium retention because of diuretic-induced neurohumoral activation may all contribute to diuretic resistance with insufficient natriuresis ${ }^{3-5}$. Consequently, it remains very difficult in clinical practice to predict how an individual patient with decompensated HF will respond to diuretic therapy and to appropriately choose the right dose and type of diuretics. In this study, we examined clinical, biochemical, and treatment predictors of diuretic efficacy assessed as elicited natriuresis per dose of loop diuretics administered. In addition, we evaluated whether the natriuretic response to diuretic therapy predicted changes in plasma $\mathrm{N}$-terminal of pro-B-type natriuretic peptide (NT-proBNP) levels, degree of neurohumoral activation during therapy, as well as clinical outcome.

\section{METHODS}

\section{Study design}

This prospective cohort study was carried out in a single tertiary care centre (Ziekenhuis Oost-Limburg, Genk, Belgium) between November 2011 and September 2013. The study protocol conforms to the ethical guidelines of the 1975 Declaration of Helsinki and was approved by the institutional committee on human research. Written informed consent was obtained from every patient. All authors had full access to the data and contributed to the writing of the manuscript, taking responsibility for the integrity of the data.

\section{Study population}

Consecutive patients, admitted with a primary diagnosis of decompensated HF, were screened. Patients were eligible for the study if $\geq 18$ years of age and able to give informed consent. Additionally, all of the following inclusion criteria had to be fulfilled: (1) presence of at least three signs of volume overload (oedema, ascites, jugular venous distension, rales, pulmonary vascular congestion on chest radiography); (2) plasma NT-proBNP $>1,000 \mathrm{ng} / \mathrm{L}$; and (3) left ventricular ejection fraction $\leq 45 \%$; for which a treatment strategy with intravenous loop diuretics was planned. Exclusion criteria were: (1) administration of intravenous diuretics before study inclusion; (2) mechanical ventilation; (3) inotropic or vasopressor support; (4) concurrent diagnosis of an acute coronary syndrome; (5) renal replacement therapy; or (6) ventricular assist devices, including the use of an intra-aortic balloon pump, at any time point during the study period.

\section{Laboratory measurements}

A baseline venous blood sample was obtained on admission before initiation of diuretic therapy, with repeated samples acquired in the morning of the next three days. Plasma NT-proBNP levels were measured by the Roche Diagnostics assay (Roche, Rotkreuz, Switzerland). Plasma renin activity was determined using the GammaCoat $^{\star}$ radioimmunoassay (DiaSorin, Sallugia, Italy). Plasma aldosterone levels were assessed by the Aldosterone Maia radioimmunoassay (Adaltis, Rome, Italy). Urine was collected during three consecutive 24 -h intervals. The first collection started together with the first administration of intravenous loop diuretics. In patients in whom difficulties with the collections were anticipated, a bladder catheter was placed to minimize urine loss.

\section{Diuretic treatment}

During the 72-h study period, loop diuretics were administered under a standard protocol as intravenous boluses of bumetanide. The initial dose was double of the patient's daily oral dose, with $1 \mathrm{mg}$ of bumetanide considered equivalent to $40 \mathrm{mg}$ of furosemide. In loop diuretic naive patients, a dose of $1 \mathrm{mg}$ was used. On morning rounds of the next three days, patients were independently evaluated by two dedicated HF specialists involved in the study, blinded to plasma NT-proBNP, plasma renin activity and plasma aldosterone measurements (M.D. \& W.M.). Based on bedside information, they decided together whether the patient was still volume overloaded. Patients with a jugular venous pressure $<8 \mathrm{mmHg}$, no orthopnoea, no rales on pulmonary auscultation and no oedema were considered to have reached a euvolemic state, after which they were switched to oral therapy and results were censored. In patients with persistent volume overload and low urine output $(<1,500 \mathrm{~mL})$, the dose of loop diuretics was doubled. All patients received a diet low in salt $(<3 \mathrm{~g}$ daily) and were instructed to limit total fluid intake to $1.5 \mathrm{~L}$. To limit occurrence of diuretic resistance, there was a strong emphasis on combinational diuretic therapy. Oral chlorthalidone - a thiazide-type diuretic - was preferentially added once daily at a dose of $50 \mathrm{mg}$ in patients with an estimated glomerular filtration rate $<40 \mathrm{~mL} / \mathrm{min} / 1.73 \mathrm{~m}^{2}$. Additionally, it was recommended that patients with a serum urea/Cr ratio $>50$ received oral acetazolamide $-\mathrm{a}$ carbonic anhydrase inhibitor - at a daily dose of $250 \mathrm{mg}$. Finally, as combination therapy with potassium-wasting diuretics increases the risk of hypokalaemia, all patients received once daily oral spironolactone at a dose of $25 \mathrm{mg}$, unless serum potassium levels were $>5.0 \mathrm{mmol} / \mathrm{L}$. All oral diuretics, including spironolactone, were administered in the morning, $1 \mathrm{~h}$ before the intravenous 
bumetanide dose. There was no pre-specified treatment strategy for patients who presented with hyponatraemia.

\section{Natriuretic response to loop diuretic therapy}

Total natriuresis $(\mathrm{mmol} / 24 \mathrm{~h})$ was calculated for each separate $24 \mathrm{~h}$-interval in every patient and loop diuretic efficacy was calculated as the ratio of total natriuresis over loop diuretic dose administered ( $\mathrm{mmol} / \mathrm{mg}$ bumetanide). Clinical, biochemical, and treatment predictors of the natriuretic response to diuretic therapy were assessed using all available 24-h urine collections. Subsequently, patients were stratified into tertiles according to their natriuretic response over the complete 72 -h study period.

\section{Decongestion, neurohumoral activation and clinical outcome}

Decongestion was assessed by the change in plasma NT-proBNP levels after $72 \mathrm{~h}$ compared to baseline. Peak neurohumoral activation during diuretic therapy was quantified as the highest of three daily values for plasma aldosterone concentration and plasma renin activity during the 72-h study period. Mortality and HF readmission data were prospectively collected by chart review. Patients who were lost to follow-up were contacted by phone to assess their vital status. HF readmissions were pre-specified as hospital admissions because of signs and/or symptoms of congestion and/or low cardiac output, during which intravenous diuretics, inotropes and/ or vasodilators were administered.

\section{Statistical analysis}

Continuous variables were expressed as mean \pm standard deviation (SD), if normally distributed, or otherwise by median (interquartile range, IQR), and compared using the Student's $t$-test or Mann-Whitney $U$ test, as appropriate. Normality was assessed by the Shapiro-Wilk statistic. Categorical data were expressed as percentages and compared by the Pearson's $X^{2}$-test. Univariate analysis was used to search for possible predictors of the natriuretic response to diuretic therapy. Predictors with $P$-value $<0.100$ were withheld in the final multivariate model, which also included the day of treatment as a random factor. Tertiles of loop diuretic responsiveness were compared by the Kruskal-Wallis $H$ test. Cumulative, actuarial survival rates were calculated according to the KaplanMeier method with the log-rank test used for comparison among tertiles of natriuretic response to diuretic therapy. To test natriuretic response as a continuous variable and correct for baseline renal function as a covariate, the Cox proportional hazards model was used to calculate a hazard ratio (HR) with corresponding 95\% confidence interval
(95\%CI) per SD change in natriuretic response. Statistical significance was always set at a 2-tailed probability level of $<0.05$. All statistics were performed using $\mathrm{IBM}^{\oplus} \mathrm{SPSS}^{\star}$ (version 22.0 for Windows).

\section{RESULTS}

\section{Study population}

Fifty-four patients were included in the study. Their baseline characteristics are presented in table 1. Preceding study inclusion, patients took a median (IQR) daily maintenance dose of $1 \mathrm{mg}$ (0-2 mg) oral bumetanide. Mean \pm SD admission estimated glomerular filtration rate (eGFR) and median (IQR) plasma NT-proBNP levels were $56 \pm 25 \mathrm{~mL} / \mathrm{min} / 1.73 \mathrm{~m}^{2}$ and $4,119 \mathrm{ng} / \mathrm{L}$ (2,257-9,264 ng/L), respectively. Other laboratory results are presented in table 2 . Nine patients (17\%) presented with serum sodium levels $<135 \mathrm{mmol} / \mathrm{L}$. Hyponatraemia was mild in all cases $(129-134 \mathrm{mmol} / \mathrm{L})$.

\section{Diuretic treatment}

Twenty-four patients (44\%), 30 (55\%) and 44 (81\%) reached a euvolemic state after $24 \mathrm{~h}, 48 \mathrm{~h}$, and $72 \mathrm{~h}$, respectively, and were switched to oral loop diuretics. The

Table 1 Baseline characteristics of the study population

$\begin{array}{lc}\text { Age (years) } & 67 \pm 13 \\ \text { Gender } & \\ \text { Male } & 74 \% \\ \quad \text { Female } & 26 \% \\ \text { New York Heart Association functional class } & \\ \text { II } & 16 \% \\ \text { III } & 54 \% \\ \text { IV } & 30 \% \\ \text { Left ventricular ejection fraction (\%) } & 24 \pm 10 \\ \text { Heart rate (bpm) } & 81 \pm 19 \\ \text { Systolic blood pressure (mmHg) } & 128 \pm 25 \\ \text { Diastolic blood pressure (mmHg) } & 71 \pm 16 \\ \text { Body mass index (kg/m }{ }^{2} \text { ) } & 29 \pm 6 \\ \text { Ischaemic heart disease } & 54 \% \\ \text { Diabetes mellitus } & 39 \% \\ \text { Chronic obstructive pulmonary disease } & 26 \% \\ \text { Medication use } & \\ \text { Renin-angiotensin system blocker } & 50 \% \\ \text { Beta blocker } & 70 \% \\ \text { Mineralocorticoid receptor antagonist } & 43 \% \\ \text { Digoxin } & 11 \% \\ \text { Loop diuretic } & 63 \%\end{array}$


median (IQR) dose of bumetanide administered was $2 \mathrm{mg}$ (1-3 mg) during the first and $1 \mathrm{mg}$ (1-2 $\mathrm{mg}$ ) during the second/third $24 \mathrm{~h}$-interval, respectively. The percentage of patients receiving oral spironolactone was $93 \%, 96 \%$ and $92 \%$ during the same 24 -h intervals, respectively.

Table 2 Baseline laboratory values of the study population

$\begin{array}{lc}\text { Haematocrit (\%) } & 37.6 \pm 6.1 \\ \text { Serum sodium (mmol/L) } & 139 \pm 4 \\ \text { Serum potassium (mmol/L } & 4.16 \pm 0.51 \\ \text { Serum chloride (mmol/L) } & 101 \pm 5 \\ \text { Serum bicarbonate }(\mathrm{mmol} / \mathrm{L}) & 24.9 \pm 3.8 \\ \text { Serum creatinine }(\mathrm{mg} / \mathrm{dL}) & 1.19(0.96-1.90) \\ \text { Estimated glomerular filtration rate }\left(\mathrm{mL} / \mathrm{min} / 1.73 \mathrm{~m}^{2}\right) & 56 \pm 25 \\ \text { Serum urea }(\mathrm{mg} / \mathrm{dL}) & 59(42-84) \\ \text { Serum urate }(\mathrm{mg} / \mathrm{dL}) & 7.89 \pm 2.66 \\ \text { Troponin T (ng/L) } & 35(24-59) \\ \text { C-reactive protein }(\mathrm{mg} / \mathrm{L}) & 5(3-16) \\ \text { HbA1c }(\%) & 6.1(5.7-6.6) \\ \text { Plasma NT-proBNP }(\mathrm{ng} / \mathrm{L}) & 4,119(2,257-9,264) \\ \text { Plasma renin activity }(\mathrm{ng} / \mathrm{mL} / \mathrm{h}) & 1.4(0.8-5.9) \\ \text { Plasma aldosterone }(\mathrm{ng} / \mathrm{L}) & 186(135-289)\end{array}$

Thirty-seven patients (69\%) received combination diuretic therapy. Chlorthalidone without acetazolamide was used in $17 \%, 20 \%$ and $17 \%$; acetazolamide without chlorthalidone in $30 \%, 27 \%$ and $17 \%$; and both agents together in $15 \%, 13 \%$ and $25 \%$, during the first, second and third $24-\mathrm{h}$ interval, respectively. Baseline renal function and natriuretic response according to the different diuretic schedules are presented in table 3. Additionally, vasodilators were used in most patients (93\%) if normo- or hypertensive and treatment with renin-angiotensin system antagonists and beta blockers was continued during the study.

\section{Natriuretic response to loop diuretic therapy}

Median(IQR) natriuresis was $146 \mathrm{mmol}(76-206 \mathrm{mmol})$, $74 \mathrm{mmol}(37-167 \mathrm{mmol})$ and $74 \mathrm{mmol}(53-134 \mathrm{mmol})$ per mg bumetanide during the first, second and third 24-h interval, respectively. Based on all available $24 \mathrm{~h}$ urinary collections (one collection was missing because of a sampling error), predictors of the natriuretic response to diuretic therapy were assessed (univariate analysis as Supplemental Data). Nine possible predictors were withheld in the final multivariate model (table 4).

Table 3 Baseline renal function and natriuretic response according to the diuretic schedule

\begin{tabular}{|c|c|c|c|c|}
\hline & Bumetanide only & $\begin{array}{l}\text { Bumetanide/ } \\
\text { acetazolamide }\end{array}$ & $\begin{array}{l}\text { Bumetanide/ } \\
\text { chlorthalidone }\end{array}$ & $\begin{array}{l}\text { Bumetanide/ } \\
\text { acetazolamide/ } \\
\text { chlorthalidone }\end{array}$ \\
\hline Baseline serum urea (mg/dl) & $50(34-59)$ & $52(40-77)$ & $82(55-145)$ & $66(57-130)$ \\
\hline Baseline serum creatinine (mg/dl) & $1.12(0.96-1.26)$ & $1.12(0.96-1.49)$ & $1.59(1.49-2.93)$ & $1.49(1.09-2.30)$ \\
\hline Baseline eGFR $\left(\mathrm{ml} / \mathrm{min} / 1.73 \mathrm{~m}^{2}\right)$ & $65 \pm 23$ & $64 \pm 25$ & $40 \pm 24$ & $46 \pm 19$ \\
\hline Natriuresis $0-24 \mathrm{~h}$ (mmol) & $343(153-467)$ & $237(161-248)$ & $170(109-181)$ & $312(248-363)$ \\
\hline Natriuresis 24-48 h (mmol) & $122(25-143)$ & $96(75-113)$ & $69(36-116)$ & $123(78-247)$ \\
\hline Natriuresis 48-72 h (mmol) & $93(45-139)$ & $153(123-190)$ & $92(71-136)$ & $113(59-200)$ \\
\hline
\end{tabular}

eGFR: estimated glomerular filtration rate.

Table 4 Predictors of the natriuretic response to diuretic therapy

$\begin{array}{lccc}\text { Predictor } & \boldsymbol{\beta} & \text { S.E. } & \text { P-value } \\ \text { Age } & -19.872 & 12.632 & 0.119 \\ \text { Left ventricular ejection fraction } & 8.945 & 11.607 & 0.443 \\ \text { Diastolic blood pressure } & 23.048 & 10.788 & 0.036 \\ \text { Maintenance dose of oral loop diuretics } & 17.132 & 11.845 & 0.152 \\ \text { Serum creatinine } & -10.992 & 14.715 & 0.457 \\ \text { Serum urea } & 16.471 & 15.454 & 0.290 \\ \text { HbA1c } & -24.139 & 12.234 & 0.052 \\ \text { Plasma aldosterone } & -25.722 & 11.560 & 0.029 \\ \text { Combination diuretic treatment with acetazolamide } & 103.241 & 40.962 & 0.014\end{array}$

Final multivariate analysis with the day of treatment included as random factor and $\beta$ expressed as the change in natriuresis per mg bumetanide for a given standard deviation change in the respective predictor.

S.E.: standard error 
Diastolic blood pressure $(\beta=23.048 \pm 10.788$; $P$-value $=$ $0.036)$, plasma aldosterone $(\beta=-25.722 \pm 11.560 ; P$-value $=$ $0.029)$ and combination therapy with acetazolamide $(\beta=103.241 \pm 40.962 ; P$-value $=0.014)$ emerged as independent predictors of the natriuretic response to loop diuretic therapy.

\section{Loop diuretic efficacy, decongestion and neurohumoral activation}

Over the entire 72 -h study period, 18 patients had a loop diuretic efficacy of $22-90 \mathrm{mmol}$ sodium/ mg bumetanide (tertile 1), $92-157 \mathrm{mmol}$ sodium/ $\mathrm{mg}$ bumetanide (tertile 2) and 161-629 mmol sodium/ mg bumetanide (tertile 3 ) each. Natriuresis during consecutive 24-h intervals, stratified according to tertiles of loop diuretic efficacy, is presented in figure 1. Overall, patients had a relative decrease in plasma NT-proBNP levels equal to $38 \%$ (13-66\%). Tertiles of increasing natriuretic response to diuretic therapy were associated

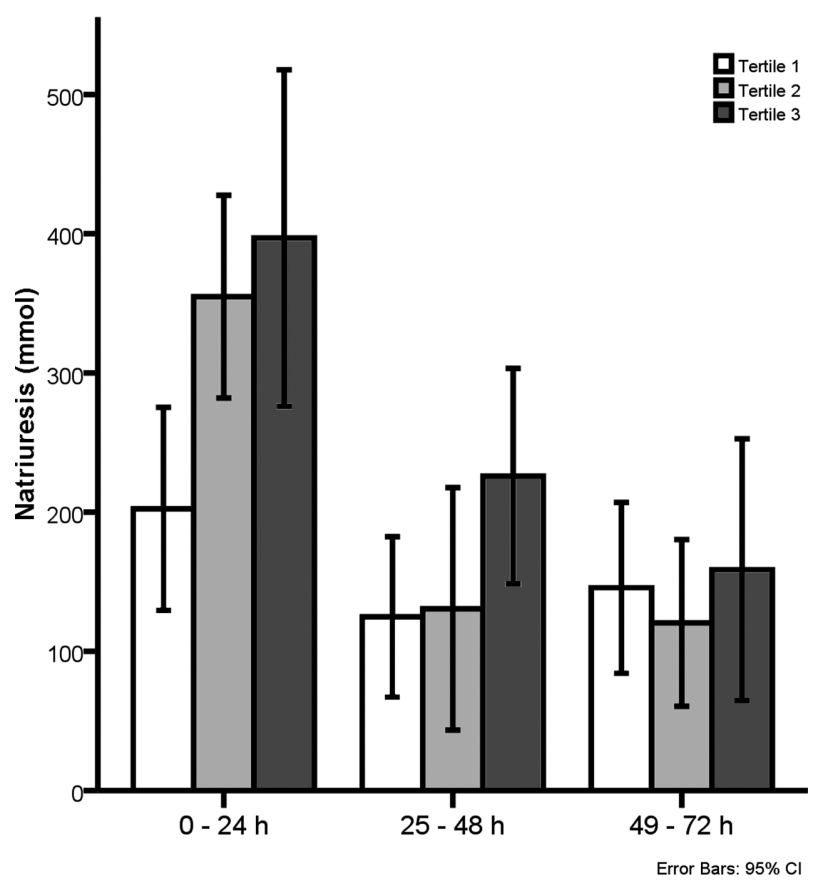

Fig. 1 Natriuresis during consecutive 24-h intervals according to tertiles of loop diuretic efficacy. with significantly greater plasma NT-proBNP decreases $(P$-value $=0.025$; figure $2 \mathrm{~A})$. On the contrary, peak neurohumoral activation, illustrated by plasma aldosterone levels $(P$-value $=0.013$; figure $2 \mathrm{~B})$ and plasma renin activity $(P$-value $=0.033$; figure $2 \mathrm{C})$, was significantly more pronounced in patients with a weaker natriuretic response.

\section{Loop diuretic efficacy and renal function}

Baseline eGFR was significantly lower in patients with a lower natriuretic response to diuretics $(42 \pm 20$ versus $57 \pm 26$ versus $64 \pm 22 \mathrm{~mL} / \mathrm{min} / 1.73 \mathrm{~m}^{2}$ for tertiles, respectively; $P$-value $=0.027)$. Creatinine clearance decreased non-significantly from the first to third $24-\mathrm{h}$ interval $\left(51 \pm 30\right.$ versus $46 \pm 26 \mathrm{~mL} / \mathrm{min} / 1.73 \mathrm{~m}^{2}$, respectively; $P$-value $=0.076$ ), with no significant change among any tertile of loop diuretic efficacy.

\section{Incidence of hypo- and hyperkalaemia}

Four patients $(7 \%)$ had transient hyperkalaemia $>5 \mathrm{mmol} / \mathrm{L}$ (one patient $>6 \mathrm{mmol} / \mathrm{L}$ ), which was spontaneously corrected in all cases after reducing or stopping spironolactone. In contrast, hypokalaemia (serum potassium level $<3.5 \mathrm{mmol} / \mathrm{L}$ ) was more frequent and occurred in 12 patients (22\%). All cases were mild an easily corrected by intravenous potassium supplements. No malignant ventricular arrhythmias were observed.

\section{Clinical outcome}

During 188 (46-386) days of follow-up, 8 patients died (15\%), 14 were admitted for decompensated HF (26\%), while 35 (65\%) had an event-free survival. Patients with a stronger natriuretic response to diuretic therapy had a better clinical outcome, free from allcause mortality or HF readmission $(P$-value $=0.010$; figure 3). Even after correction for baseline eGFR, the adjusted HR (95\% CI) for all-cause mortality or HF readmission was equal to $0.40(0.16-0.98)$ per SD increase in natriuretic response to loop diuretic therapy $(P$-value $=0.045)$.
Fig. 2 Change in (A) plasma NT-proBNP levels and peak neurohumoral activation, assessed by (B) plasma aldosterone levels and (C) plasma renin activity, according to tertiles of the natriuretic response to diuretic therapy.
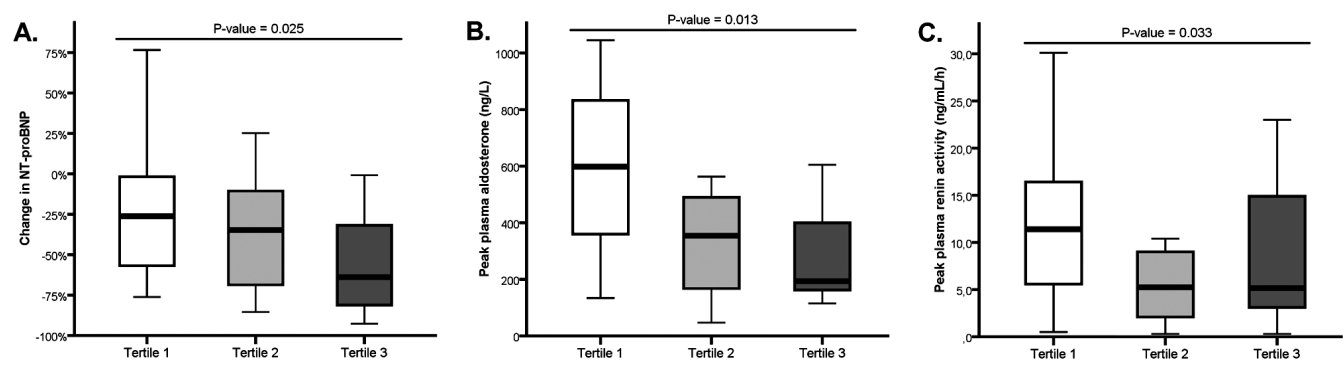


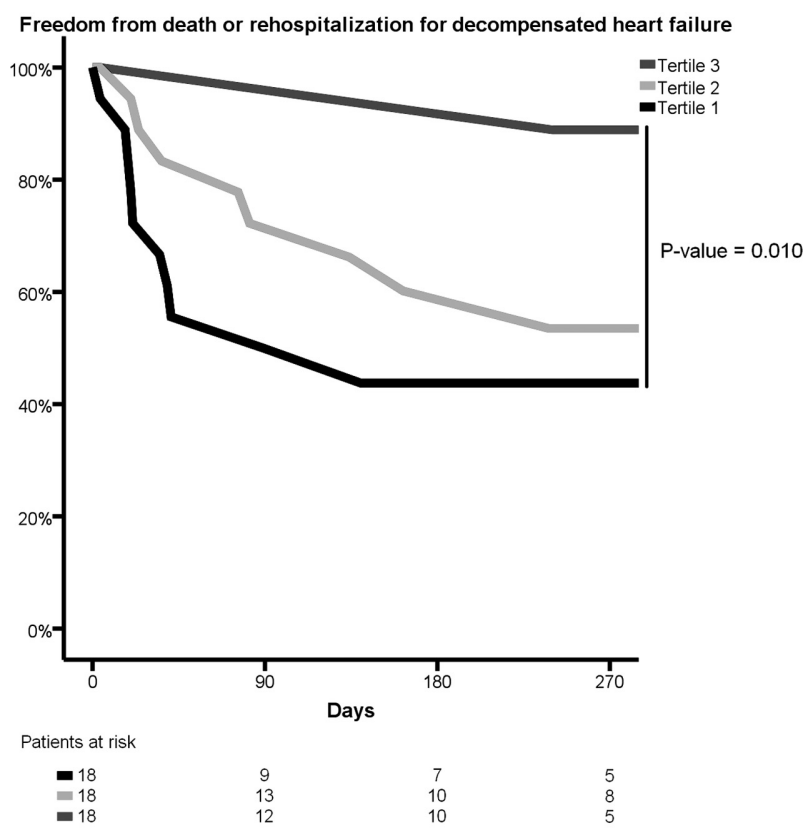

Fig. 3 Freedom from death or rehospitalization for decompensated heart failure according to tertiles of the natriuretic response to diuretic therapy.

\section{DISCUSSION}

Although directly representing the pharmacodynamic effect of diuretics in decompensated HF, natriuresis is not routinely assessed and its determinants remain insufficiently elucidated, especially in the contemporary era of HF treatment. The key finding of this prospective cohort study in patients with decompensated HF, low ejection fraction and volume overload is that a stronger natriuretic response to diuretics is associated with a larger decrease in plasma NT-proBNP levels, less neurohumoral activation, and better clinical outcome. Our results suggest that the natriuretic response to diuretics might be used as a surrogate marker of effective decongestive treatment and long-term outcomes in future studies of decompensated HF. Several other findings are noteworthy as they provide further insight into complex cardio-renal interactions in decompensated HF: (1) lower diastolic blood pressure and higher plasma aldosterone levels were associated with a weaker natriuretic response to diuretics; (2) inhibition of sodium reabsorption in the proximal tubules with acetazolamide increased the natriuretic response to loop diuretics; (3) ubiquitous upfront use of spironolactone in patients presenting with decompensated HF was safe, with a low incidence of hyperkalaemia (7\%), which was generally mild and did not lead to malignant ventricular arrhythmias.
Different studies have repeatedly shown that achieving effective decongestion in patients with decompensated $\mathrm{HF}$ is associated with improved survival and less readmissions, even if serum creatinine levels rise transiently ${ }^{6-8}$. This arguably calls for more direct measurements of decongestion in future HF studies. Intriguingly, a relationship between loop diuretic efficacy - defined as urine output over loop diuretic dose - and all-cause mortality has recently been described and validated in two independent prospective cohorts, encompassing more than thousand patients together'. Even after adjusting for in-hospital diuretic dose, urine output and baseline characteristics, poor loop diuretic efficacy remained associated with worse survival. Importantly, the fact that diuretic efficacy incorporates aspects of both cardiac and renal function, makes it an attractive parameter to represent decongestion in decompensated HF. In a more mechanistic study, which assessed urinary sodium over urinary furosemide concentrations during continuous intravenous administration, our group has reported the incremental value of natriuresis over classic measurements of kidney function ${ }^{10}$. In that study of 52 decompensated HF patients, poor natriuretic response to furosemide was associated with low urine output, worsening renal function and adverse clinical outcome ${ }^{10}$. The current study reaffirms the concept of diuretic efficacy as an important prognostic marker in decompensated HF, providing more insight in its determinants. Low diastolic blood pressure was associated with poor natriuresis. Indeed, it has been reported that hypotensive episodes during decongestive treatment in decompensated HF are associated with a drop in glomerular filtration rate, as the arterial blood pressure is a critical determinant of glomerular capillary pressure $^{11,12}$. In addition, higher plasma aldosterone levels, representing neurohumoral activation and aldosterone breakthrough were also predictive of a poor natriuretic response to diuretics.

A surprising finding was that combination therapy with acetazolamide - a carbonic anhydrase inhibitor that reduces sodium reabsorption in the proximal tubules - was able to significantly increase loop diuretic efficacy. In multivariate analysis corrected for age, ejection fraction, diastolic blood pressure, plasma aldosterone levels, $\mathrm{HbAl}_{\mathrm{C}}$, underlying renal function and loop diuretic dose, combination therapy with acetazolamide increased natriuresis with more than $100 \mathrm{mmol}$ per $\mathrm{mg}$ bumetanide, which would be the equivalent of nearly $6 \mathrm{~g}$ salt. This finding should be considered hypothesis-generating as acetazolamide administration was not randomized, but there might be some pathophysiological rationale. Indeed, plasma filtration fraction is often increased in patients with decompensated HF, resulting in lower hydrostatic but higher colloid osmotic pressure 
in peritubular capillaries, driving sodium reabsorption in the proximal tubules and potentially contributing to loop diuretic resistance ${ }^{5,13-15}$. Only a few small-scale and largely outdated studies have evaluated combination therapy with acetazolamide in decompensated HF, yet they did show promising results to break loop diuretic resistance ${ }^{16,17}$. Furthermore, it warrants notion that we specifically targeted patients with high blood urea nitrogen levels for treatment with acetazolamide, a population that has been characterized previously as particularly prone to poor outcomes with high-dose loop diuretics ${ }^{18}$.

Finally, our study offers some insightful data about the feasibility and safety of upfront therapy with mineralocorticoid receptor antagonists in decompensated HF. The benefits of mineralocorticoid receptor antagonists in ambulatory HF patients are now widely established, with such treatment reducing mortality and readmissions in patients with advanced as well as paucisymptomatic $\mathrm{HF}^{19,20}$. Importantly, aldosterone breakthrough occurs in many HF patients despite treatment with an angiotensin-converting enzyme inhibitor at an adequate dose $^{21}$. Although decongestive treatment with loop diuretics further boosts systemic and intrarenal aldosterone production, there is a lack of data regarding the use of mineralocorticoid receptor antagonists in decompensated $\mathrm{HF}^{3}$. Despite including sick patients with impaired renal function, more than $90 \%$ of patients in our study received spironolactone as add-on therapy during treatment of volume overload without major adverse events. Spironolactone was maintained at the time of discharge in $84 \%$, with $86 \%$ of patients receiving it at the first follow-up appointment after discharge.

\section{STUDY LIMITATIONS}

Some limitations should be acknowledged when interpreting the study results. First, this was a single-centre study with limited sample size. Therefore, our results should be considered exploratory and hypothesis-generating. Second, the population studied was a selected group of sick patients with decompensated HF, illustrated by a median plasma NT-proBNP level of $4,119 \mathrm{ng} / \mathrm{L}$. Therefore, our findings are specifically applicable to this vulnerable population. Third, although the study provided a standardized protocol for the administration of diuretics, their use was not randomized with a control group, which resulted, for instance, in sicker patients being preferentially co-treated with chlorthalidone and acetazolamide. The true value of these diuretics in improving the natriuretic response to loop diuretics can therefore only be evaluated in the context of a randomized clinical trial. To assess the potential value of acetazolamide to increase diuretic efficiency in patients at high risk for cardio-renal syndrome, recently such a trial was started at our centre (NCT01973335).

\section{CONCLUSION}

Greater loop diuretic efficacy, assessed as natriuresis per loop diuretic dose, is associated with more thorough decongestion and less neurohumoral activation in decompensated HF patients with low ejection fraction and volume overload, when compared to patients with a weaker natriuretic response. Moreover, natriuretic response is a strong predictor of clinical outcome, independently of underlying renal function. Balanced use of combinational diuretic therapy, in particular with acetazolamide, might enhance natriuresis, precluding the need for high-dose loop diuretics in decompensated HF.

\section{ACKNOWLEDGEMENTS}

Special thanks to the nursing team of the heart failure intensive care unit of Ziekenhuis Oost-Limburg for their invaluable help in acquiring the data. Also thanks to Carmen Reynders for her help with the laboratory analyses.

\section{FINANCIAL SUPPORT}

Frederik Verbrugge (11L8214N) and Philippe Bertrand $(11 \mathrm{~N} 7214 \mathrm{~N})$ are supported by a Ph.D. fellowship of the Research Foundation - Flanders (FWO). Frederik Verbrugge, Philippe Bertrand, Petra Nijst, Joseph Dens, Pieter Vandervoort and Wilfried Mullens are researchers for the Limburg Clinical Research Program (LCRP) UHasselt-ZOL-Jessa, supported by the foundation Limburg Sterk Merk (LSM), Hasselt University, Ziekenhuis Oost-Limburg and Jessa Hospital.

CONFLICT OF INTEREST: none declared. 
SUPPLEMENTAL DATA Predictors of the natriuretic response to diuretic therapy on univariate analysis

\begin{tabular}{|c|c|c|c|}
\hline Parameter & $\beta$ & S.E. & $P$-value \\
\hline Age (years) & -2.685 & 0.816 & 0.001 \\
\hline Female gender & 19.015 & 24.141 & 0.433 \\
\hline New York Heart Association functional class (per class increase) & -9.424 & 15.138 & 0.535 \\
\hline Left ventricular ejection fraction (\%) & -2.147 & 1.024 & 0.038 \\
\hline Heart rate (bpm) & 0.694 & 0.611 & 0.258 \\
\hline Systolic blood pressure $(\mathrm{mmHg})$ & 0.267 & 0.438 & 0.543 \\
\hline Diastolic blood pressure $(\mathrm{mmHg})$ & 2.509 & 0.634 & $<0.001$ \\
\hline Body mass index $\left(\mathrm{kg} / \mathrm{m}^{2}\right)$ & -1.756 & 1.712 & 0.307 \\
\hline Ischaemic heart disease & -22.199 & 20.767 & 0.288 \\
\hline Chronic obstructive pulmonary disease & -37.222 & 22.831 & 0.106 \\
\hline Maintenance therapy with renin-angiotensin system blocker & 33.632 & 20.778 & 0.109 \\
\hline Maintenance therapy with beta blocker & -22.980 & 21.863 & 0.296 \\
\hline Maintenance therapy with mineralocorticoid receptor blocker & -30.133 & 20.829 & 0.151 \\
\hline Maintenance therapy with digoxin & -47.919 & 30.436 & 0.118 \\
\hline Maintenance dose of oral loop diuretics ( $\mathrm{mg}$ bumetanide equivalents*) & -23.132 & 7.917 & 0.004 \\
\hline Haematocrit (\%) & 1.674 & 1.622 & 0.304 \\
\hline Serum sodium (mmol/L) & 0.716 & 2.896 & 0.805 \\
\hline Serum potassium (mmol/L) & 16.607 & 19.950 & 0.407 \\
\hline Serum chloride (mmol/L) & 3.513 & 2.244 & 0.121 \\
\hline Serum bicarbonate (mmol/L) & -3.506 & 2.496 & 0.163 \\
\hline Serum creatinine $(\mathrm{mg} / \mathrm{dL})$ & -36.271 & 13.373 & 0.008 \\
\hline Serum urea $(\mathrm{mg} / \mathrm{dL})$ & -0.537 & 0.198 & 0.008 \\
\hline Serum urate $(\mathrm{mg} / \mathrm{dL})$ & -1.609 & 4.206 & 0.703 \\
\hline Troponin T (ng/L) & -0.270 & 0.187 & 0.152 \\
\hline C-reactive protein (mg/L) & 0.466 & 0.561 & 0.408 \\
\hline $\operatorname{HbA1C}(\%)$ & -39.108 & 11.084 & 0.001 \\
\hline Plasma NT-proBNP (ng/L) & 0 & 0.001 & 0.862 \\
\hline Plasma renin activity $(\mathrm{ng} / \mathrm{mL} / \mathrm{h})$ & -1.685 & 1.383 & 0.226 \\
\hline Plasma aldosterone (ng/L) & -0.107 & 0.048 & 0.029 \\
\hline Combination diuretic treatment with chlorthalidone & -17.056 & 21.914 & 0.438 \\
\hline Combination diuretic treatment with acetazolamide & 52.191 & 20.348 & 0.012 \\
\hline
\end{tabular}




\section{REFERENCES}

1. Adams KF, Jr., Fonarow GC, Emerman CL, LeJemtel TH, Costanzo MR, Abraham WT Berkowitz RL, Galvao M, Horton DP; ADHERE Scientific Advisory Committee and Investigators. Characteristics and outcomes of patients hospitalized for heart failure in the United States: rationale, design, and preliminary observations from the first 100,000 cases in the Acute Decompensated Heart Failure National Registry (ADHERE). Am Heart J 2005; 149: 209-16.

2. Fonarow GC, Corday E; ADHERE Scientific Advisory Committee. Overview of acutely decompensated congestive heart failure (ADHF): a report from the ADHERE registry. Heart Fail Rev 2004; 9: 179-85.

3. Krämer BK, Schweda F, Riegger GA. Diuretic treatment and diuretic resistance in heart failure. Am J Med 1999; 106: 90-6.

4. Verbrugge FH, Dupont M, Steels $P$, Grieten $L$, Malbrain M, Tang WH, Mullens W. Abdominal contributions to cardiorenal dysfunction in congestive heart failure.

J Am Coll Cardiol 2013; 62: 485-95.

5. Verbrugge FH, Dupont M, Steels P, Grieten L, Swennen Q, Tang WH, Mullens W. The kidney in congestive heart failure: 'are natriuresis, sodium, and diuretics really the good, the bad and the ugly?'.

Eur J Heart Fail 2014; 16: 133-42.

6. Costanzo MR, Guglin ME, Saltzberg MT, Jessup ML, Bart BA, Teerlink JR, Jaski BE, Fang JC, Feller ED, Haas GJ, Anderson AS, Schollmeyer MP, Sobotka PA; UNLOAD Tria Investigators. Ultrafiltration versus intravenous diuretics for patients hospitalized for acute decompensated heart failure. J Am Coll Cardiol 2007; 49: 675-83.

7. Testani JM, Chen J, McCauley BD, Kimmel SE, Shannon RP. Potential effects of aggressive decongestion during the treatment of decompensated heart failure on renal function and survival.

Circulation 2010; 122: 265-72.

8. Metra M, Davison B, Bettari L, Sun $\mathrm{H}$, Edwards C, Lazzarini V, Piovanelli B, Carubelli V, Bugatti S, Lombardi C, Cotter G, Dei Cas L. Is worsening renal function an ominous prognostic sign in patients with acute heart failure? The role of congestion and its interaction with renal function. Circ Heart Fail 2012; 5: 54-62.

9. Testani JM, Brisco MA, Turner JM, Spatz ES, Bellumkonda L, Parikh CR, Tang WH. Loop diuretic efficiency: a metric of diuretic responsiveness with prognostic importance in acute decompensated heart failure. Circ Heart failure 2014; 7: 261-70.

10. Singh D, Shrestha K, Testani JM, Verbrugge FH, Dupont M, Mullens W, Tang WH. Insufficient natriuretic response to continuous intravenous furosemide is associated with poor long-term outcomes in acute decompensated heart failure. J Card Fail 2014; 20: 392-9.

11. Testani JM, Coca SG, McCauley BD, Shannon RP, Kimmel SE. Impact of changes in blood pressure during the treatment of acute decompensated heart failure on renal and clinical outcomes. Eur J Heart Fail 2011; 13: 877-84.

12. Dupont M, Mullens W, Finucan M, Taylor DO, Starling RC, Tang WH. Determinants of dynamic changes in serum creatinine in acute decompensated heart failure: the importance of blood pressure reduction during treatment.

Eur J Heart Fail 2013; 15: 433-40.

13. Lewy JE, Windhager EE.

Peritubular control of proximal tubular fluid reabsorption in the rat kidney. Am J Physiol 1968; 214: 943-54.

14. Grandchamp A, Boulpaep EL. Pressure control of sodium reabsorption and intercellular backflux across proximal kidney tubule. J Clin Invest 1974; 54: 69-82.

15. Smilde TD, Damman $K$, van der Harst $P$, Navis G, Westenbrink BD, Voors AA, Boomsma F, van Veldhuisen DJ, Hillege HL. Differential associations between renal function and "modifiable" risk factors in patients with chronic heart failure. Clin Res Cardiol 2009; 98: 121-9.

16. Knauf $H$, Mutschler E. Sequential nephron blockade breaks resistance to diuretics in edematous states.

J Cardiovasc Pharmacol 1997; 29: 367-72.

17. Khan MI. Treatment of refractory congestive heart failure and normokalemic hypochloremic alkalosis with acetazolamide and spironolactone. Can Med Assoc J 1980; 123: 883-7.

18. Testani JM, Cappola TP, Brensinger CM, Shannon RP, Kimmel SE. Interaction between loop diuretic-associated mortality and blood urea nitrogen concentration in chronic heart failure. J Am Coll Cardiol 2011; 58: 375-82.

19. Pitt B, Zannad F, Remme WJ, Cody R, Castaigne A, Perez A, Palensky J, Wittes J. The effect of spironolactone on morbidity and mortality in patients with severe heart failure. Randomized Aldactone Evaluation Study Investigators. NEngl J Med 1999; 341: 709-17.

20. Zannad F, McMurray JJ, Krum H, van Veldhuisen DJ, Swedberg K, Shi H, Vincent J, Pocock SJ, Pitt B. Eplerenone in patients with systolic heart failure and mild symptoms. N Engl J Med 2011; 364: 11-21.

21. Tang $W H$, Vagelos $R H$, Yee $Y G$, Benedict CR, Willson $\mathrm{K}$, Liss CL, Fowler MB. Neurohormonal and clinical responses to high- versus low-dose enalapril therapy in chronic heart failure. J Am Coll Cardiol 2002; 39: 70-8. 